\title{
Suppression of the outwards-dispersing branches in hour-glass magnetic spectra induced by nanoscale phase separation in $\mathrm{La}_{2-x} \mathrm{Sr}_{x} \mathrm{CoO}_{4}$
}

\author{
H. Guo, ${ }^{1}$ Z. W. Li, ${ }^{1}$ S. Sakong, ${ }^{1}$ G. Ryu, ${ }^{1}$ L. Zhao, ${ }^{1}$ A. Piovano, ${ }^{2}$ W. Schmidt, ${ }^{2,3}$ M. Sprung, ${ }^{4}$ J. Strempfer, ${ }^{4}$ S. Francoual, ${ }^{4}$ \\ D. Dzhigaev, ${ }^{4}$ S. Subakti, ${ }^{1}$ Z. Hu, ${ }^{1}$ H. J. Lin, ${ }^{5}$ C. T. Chen,${ }^{5}$ H. Luetkens, ${ }^{6}$ O. Stockert, ${ }^{1}$ and A. C. Komarek ${ }^{1, *}$ \\ ${ }^{1}$ Max Planck Institute for Chemical Physics of Solids, Nöthnitzer Str. 40, D-01187 Dresden, Germany \\ ${ }^{2}$ Institut Laue-Langevin (ILL), 71 Avenue des Martyrs, F-38042 Grenoble Cedex 9, France \\ ${ }^{3}$ Jülich Centre for Neutron Science JCNS, Forschungszentrum Jülich GmbH, Outstation at ILL, 71 Avenue des Martyrs, \\ F-38042 Grenoble Cedex 9, France \\ ${ }^{4}$ Deutsches Elektronen-Synchrotron DESY, Notkestrasse 85, D-22607 Hamburg, Germany \\ ${ }^{5}$ National Synchrotron Radiation Research Center (NSRRC), 101 Hsin-Ann Road, Hsinchu 30076, Taiwan \\ ${ }^{6}$ Paul Scherrer Institut, Forschungsstr. 111, CH-5232 Villigen PSI, Switzerland
}

(Received 25 March 2019; revised manuscript received 13 June 2019; published 9 July 2019)

\begin{abstract}
Here, we reinvestigate the underlying charge and magnetic correlations in $\mathrm{La}_{2-x} \mathrm{Sr}_{x} \mathrm{CoO}_{4}$ within a combined study of muon spin relaxation $(\mu \mathrm{SR})$, x-ray photon correlation spectroscopy, synchrotron radiation singlecrystal x-ray diffraction, and neutron-scattering measurements. For $\mathrm{La}_{2-x} \mathrm{Sr}_{x} \mathrm{CoO}_{4}$ around 1/3 hole-doping, the significant charge correlations that are responsible for the emergence of hour-glass magnetic spectra are found to be slowly fluctuating checkerboard charge ordering ( $\mathrm{CBCO}$ ) correlations with an onset temperature being independent of hole-doping. This reveals a local origin of the $\mathrm{CBCO}$ correlations in the rather ionic cobaltates, thus being distinct from the more covalent nickelates. Moreover, we report the observation of a very similar temperature dependence of the intensities of in-plane high- and low-energy excitations within the $\mathrm{La}_{2-x} \mathrm{Sr}_{x} \mathrm{CoO}_{4}$ hour-glass spectrum. This observation shows that nano phase separation is distinct from conventional phase separation and that there is a coupling between nanometer-sized undoped and hole-doped islands. Based on this, it is possible to propose a microscopic scenario for the suppression of the outwards-dispersing branches in hour-glass magnetic spectra.
\end{abstract}

DOI: 10.1103/PhysRevB.100.014411

\section{INTRODUCTION}

Magnetic interactions are widely believed to play an important role for the superconducting pairing mechanism in cuprate high-temperature superconductors (HTSC). When plotted in energy-momentum space, the HTSC cuprate materials exhibit a magnetic excitation spectrum that resembles an hour-glass [1-11]. Since their initial discovery, the underlying physics of these hour-glass magnetic spectra is controversially discussed [12-15] and remains puzzling. Besides models based on Fermi-surface effects, charge-stripe models are also proposed to explain these spectra. However, even some of the most prominent features of these hour-glass spectra, like the suppression of the outwards-dispersing branches, are not yet well understood on a microscopic level.

Recently, hour-glass magnetic spectra were also observed in an isostructural but insulating reference system-the cobaltates $\mathrm{La}_{2-x} \mathrm{Sr}_{x} \mathrm{CoO}_{4}$ [16]. Due to their insulating nature, Fermi-surface effects can be neglected in these Co oxides. Hence, it was conjectured that charge stripes are responsible for the emergence of hour-glass magnetic spectra [16] and that disorder was (phenomenologically) connected to the suppression of the outwards-dispersing branches [17].

\footnotetext{
*Komarek@cpfs.mpg.de
}

However, it was shown in subsequent studies that chargestripe order (CSO) is essentially absent in these cobaltates $[18,19]$, and instead, that a short-ranged checkerboard charge-ordered (CBCO) phase emerges in these systems [18-20]. These observations were the basis for an entirely new model of hour-glass magnetic spectra-a nano phase separation model [19]. Within this nano phase separation scenario, nanometer-sized $\mathrm{La}_{2} \mathrm{CoO}_{4}$-like undoped islands and $\mathrm{La}_{1.5} \mathrm{Sr}_{0.5} \mathrm{CoO}_{4}$-like hole-rich $\mathrm{CBCO}$ regions coexist. That $\mathrm{La}_{2-x} \mathrm{Sr}_{x} \mathrm{CoO}_{4}$ samples with hour-glass spectra exhibit nanoscale-phase separation was confirmed by resonant microdiffraction measurements reporting an average domain size of CBCO regions of the order of one nanometer [19]. Supported by numerical simulations, it was shown that the high-energy magnetic excitations within the hour-glass magnetic spectrum entirely arise from spin excitations within the nanoscopic undoped islands whereas the strongest low-energy spin excitations could be assigned to the hole-rich CBCO islands [19]. Indeed, there are similarities between the upper part of the hour-glass magnetic spectrum in $\mathrm{La}_{2-x} \mathrm{Sr}_{x} \mathrm{CoO}_{4}$ and the spectrum in undoped $\mathrm{La}_{2} \mathrm{CoO}_{4}$ [21] and also similarities of the lower part of the hour-glass spectrum and half-doped $\mathrm{La}_{1.5} \mathrm{Sr}_{0.5} \mathrm{CoO}_{4}$ [22]. The major difference to conventional phase separation is that the outwards-dispersing branches of the low-energy magnetic excitations are missing (and that energies are renormalized to some extent), i.e., 
nano phase separation is different from conventional phase separation where the observation of a simple superposition of the magnetic excitation spectra of undoped and half-doped phases would be expected-without any suppression of the outwards-dispersing branches of the low-energy excitations (and without a renormalization of energies) [23].

Finally, there exist very recent studies of the charge correlations in cobaltates around 1/3 hole-doping, which can be interpreted in terms of an even more complex nano phase separation scenario, where in addition to the $\mathrm{CBCO}$ phases, some fractions of CSO phases also appear [24]. Note that nanoscale phase-separation phenomena are also recently discussed in the high-temperature superconducting cuprates [25], iron chalcogenides [26,27], and other superconductors [28].

Herein, we reinvestigate the charge correlations in these cobaltates around $\sim 1 / 3$ hole-doping using x-ray diffraction and (polarized) neutron scattering and mapping the magnetic excitation spectrum with polarized neutrons. Thus, we are able to present a comprehensive picture of the physics in these cobalt oxides including a possible microscopic scenario that would naturally explain the suppression of the outwardsdispersing branches within their hour-glass shaped magnetic excitation spectra.

\section{EXPERIMENTAL}

Single crystals of $\mathrm{La}_{2-x} \mathrm{Sr}_{x} \mathrm{CoO}_{4}(x=0,0.1,0.2,0.33$, $0.36,0.37,0.38,0.4,0.5,0.6,0.8$ ) were grown by the floating zone method as described elsewhere [18].

To determine the Co oxidation state in $\mathrm{La}_{1.63} \mathrm{Sr}_{0.37} \mathrm{CoO}_{4}$ Co- $\mathrm{L}_{2,3}$ x-ray absorption spectroscopy (XAS) spectra were measured at beamline 11 A of the National Synchrotron Radiation Research Center in Taiwan. The Co- $\mathrm{L}_{2,3}$ XAS spectra were collected at room temperature in an ultrahigh vacuum chamber $\left(10^{-10} \mathrm{mbar}\right)$ using total-electron yield mode with an energy resolution of around $0.25 \mathrm{eV}$. A CoO single crystal was measured simultaneously in a separate chamber to calibrate the photon energy with an accuracy better than $10 \mathrm{meV}$. In Fig. 1, the isotropic Co- $\mathrm{L}_{2,3} \mathrm{XAS}$ spectrum of $\mathrm{La}_{1.63} \mathrm{Sr}_{0.37} \mathrm{CoO}_{4}$ (black line) is shown together with the ones of $\mathrm{La}_{2} \mathrm{CoO}_{4}$ (green line) and $\mathrm{LaSrCoO}_{4}$ (blue line) as a $\mathrm{Co}^{2+}$ and $\mathrm{Co}^{3+}$ reference, respectively. The red and cyan lines are a superposition of the $\mathrm{Co}^{2+}$ and $\mathrm{Co}^{3+}$ reference spectra according to a formal valence of $2.37+$ and $2.38+$, respectively. The XAS spectrum of $\mathrm{La}_{1.63} \mathrm{Sr}_{0.37} \mathrm{CoO}_{4}$ is between these simulated spectra, thus, demonstrating a close to stoichiometric oxygen content of $\sim 4.0025$. For $\mathrm{La}_{1.62} \mathrm{Sr}_{0.38} \mathrm{CoO}_{4}$, the oxygen stoichiometry was determined by thermogravimetric measurements, showing an oxygen content of 3.999(21). Finally, the oxidation state of our samples is also ascertained by the magnetic incommensurabilities $2 \varepsilon_{\mathrm{mag}}$ of our $\mathrm{La}_{2-x} \mathrm{Sr}_{x} \mathrm{CoO}_{4}$ samples (observed in neutron-scattering experiments), which amount to 0.386(9) and 0.368(5) for $x=$ 0.38 and 0.37, respectively. (The $\mathrm{La}_{5 / 3} \mathrm{Sr}_{1 / 3} \mathrm{CoO}_{4}$ sample with an oxygen content of about 4.01 was already characterized in Refs. $[18,19]$.)

To study the charge correlations in these cobaltates, synchrotron-radiation single-crystal $\mathrm{x}$-ray diffraction measurements have been performed at beamline P09 at PETRA

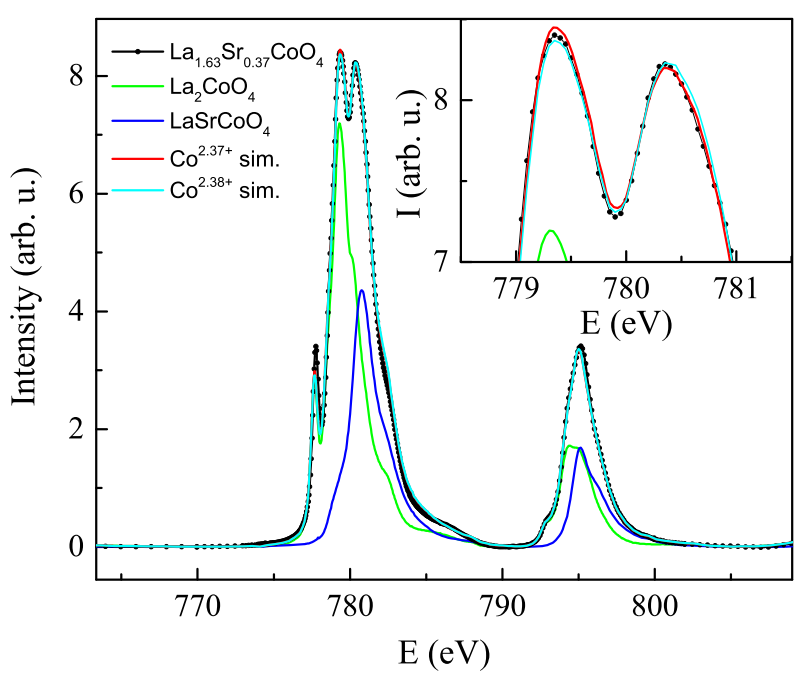

FIG. 1. XAS spectra of $\mathrm{La}_{1.63} \mathrm{Sr}_{0.37} \mathrm{CoO}_{4}$ (black) together with $\mathrm{Co}^{2+}$ and $\mathrm{Co}^{3+}$ reference spectra as well as their superposition according to a simulated $\mathrm{Co}^{2.37+}$ (red) and $\mathrm{Co}^{2.38+}$ (blue) oxidation state. The inset shows a close-up of the main peak at the $\mathrm{L}_{3}$ edge.

III, DESY. The incident photon energy was $7680 \mathrm{eV}$, which is off resonance and not sensitive to any magnetic signal.

To measure the charge and magnetic correlations in the $\mathrm{La}_{2-x} \mathrm{Sr}_{x} \mathrm{CoO}_{4}$ system, (un-)polarized neutron-scattering experiments have been performed. For the temperaturedependent and the polarized neutron-scattering experiments, two coaligned single crystals of $\mathrm{La}_{1.62} \mathrm{Sr}_{0.38} \mathrm{CoO}_{4}$ with $[100] /[010]$ orientation in the scattering plane were used. The experiments have been performed at the IN8 and IN20 thermal triple-axis spectrometers at the ILL in Grenoble, France. Double-focusing pyrolytic graphite (PG) monochromator and analyzer were used for the temperature dependent measurements at IN8 and double focusing Heusler (111) monochromator and horizontally focusing Heusler (111) analyzer were used for the polarized neutron measurements at the IN20 spectrometer. All of our experiments were performed with two PG filters to suppress higher order neutron contaminations.

$\mathrm{X}$-ray photon correlation spectroscopy (XPCS) measurements have been performed at the beamline P10 of PETRA III using $13.8 \mathrm{keV}$ x-rays (with $2 \times 2 \mu \mathrm{m}$ beam size). An EIGER 4M 2D detector with $2070 \times 2167$ pixels $(75 \mu \mathrm{m} \times$ $75 \mu \mathrm{m}$ per pixel) was used (5.05 m sample-detector distance). A series of frames with $5 \mathrm{~s}-10 \mathrm{~s}$ exposure time was taken within several hours at $Q=(0.50 .56 .5)$, and only the frames measured about one hour after the stabilization of the temperature were used for the normalized intensity autocorrelation calculation. The normalized autocorrelation function $g_{2}(t)$ is defined as

$$
g_{2}(t)=\frac{\langle I(\tau) I(t+\tau)\rangle_{\tau}}{\langle I(\tau)\rangle_{\tau}^{2}}
$$

where $I(\tau)$ and $I(t+\tau)$ are the intensities taken at time $\tau$ and $\mathrm{t}$ $+\tau$, respectively, for one pixel, and $\langle\ldots\rangle_{\tau}$ indicates an average over the pixels and time $\tau$. The obtained $g_{2}(t)$ function can be well described by the Kohlrausch-Williams-Watts (KWW) expression as

$$
g_{2}(t)=1+A \exp \left[-2(t / \tau)^{\beta}\right],
$$


where $A$ is related to the beam coherence, $\beta$ is the exponent parameter, and $\tau$ is the relaxation time.

For studying the magnetic phase diagram of the $\mathrm{La}_{2-x} \mathrm{Sr}_{x} \mathrm{CoO}_{4}$ system, muon-spin relaxation $(\mu \mathrm{SR})$ measurements have been performed on the GPS instrument at the Swiss Muon Source $\mathrm{S} \mu \mathrm{S}$, Paul Scherrer Institute, Villigen, Switzerland. Nearly $100 \%$ spin polarized muons were injected into the sample, and the decayed positrons which were ejected preferentially along the muon spin direction were accumulated by the forward and backward counters. The asymmetry is defined as $A(t)=\left[N_{F}(t)-\alpha N_{B}(t)\right] /\left[N_{F}(t)+\alpha N_{B}(t)\right]$, where $N_{F}(t)\left(N_{B}(t)\right)$ is the number of positrons arrived at the forward (backward) counter at time $t$, and the parameter $\alpha$ reflects the different counting efficiencies of the counters. The muon events are counted in the veto mode, resulting in nearly zero background. The long-range magnetic ordering onset temperature is determined either by the appearance of the internal fields, as done for the undoped parent compound $\mathrm{La}_{2} \mathrm{CoO}_{4}$, or by the reduction of the paramagnetic volume fraction obtained from weak transverse field $(30 \mathrm{G})$ measurements - the latter ones being more efficient for the determination of the magnetic transition temperatures of a larger amount of samples within the $\mathrm{La}_{2-x} \mathrm{Sr}_{x} \mathrm{CoO}_{4}$ phase diagram. The data was analyzed using the MUSRFIT package [29].

\section{RESULTS}

\section{A. Charge correlations}

In Refs. [18-20,30], it was shown that there are no apparent indications for CSO correlations in $\mathrm{La}_{2-x} \mathrm{Sr}_{x} \mathrm{CoO}_{4}$ around $1 / 3$ or 0.4 hole-doping for smaller or zero values of $L$. To clarify the origin of the recently observed signals at the tail of the checkerboard charge-ordering peaks for large values of $L$ in Ref. [24], we have also investigated the charge correlations in $\mathrm{La}_{2-x} \mathrm{Sr}_{x} \mathrm{CoO}_{4}$ around $1 / 3$ doping systematically.

\section{Neutron-scattering experiments}

First, we characterized the magnetic peaks of our $\mathrm{La}_{1.63} \mathrm{Sr}_{0.37} \mathrm{CoO}_{4}$ single crystalline sample with polarized neutrons-see Fig. 2. The magnetic incommensurability almost exactly corresponds to the hole-doping level $x$. As can be seen, there is another third, central magnetic contribution that needs to be described by another broad Gaussian peak. These observations were also made in previous measurements $[16,18,19,24]$. The presence of half-integer magnetic intensities is in agreement with the recent nano phase separation scenario [19].

For studying the charge correlations, we have performed HH7-scans similar as reported in Ref. [24]—see Fig. 3. Similar to Ref. [24], we also obtain very similar peak broadening effects or additional intensities at the shoulders of the halfinteger peaks having almost the same integrated intensities below the peak differences as in the former study [24], see Fig. 3. For comparing the volume fractions of $\mathrm{CBCO}$ and any possible CSO phases in quasi-2D systems (with weak $L$-dependence of the signal) one should take account for the entire volume under the two-dimensional peak-see inset of Fig. 3-and not only for the area under a single one-dimensional scan which is only a cut through this two-

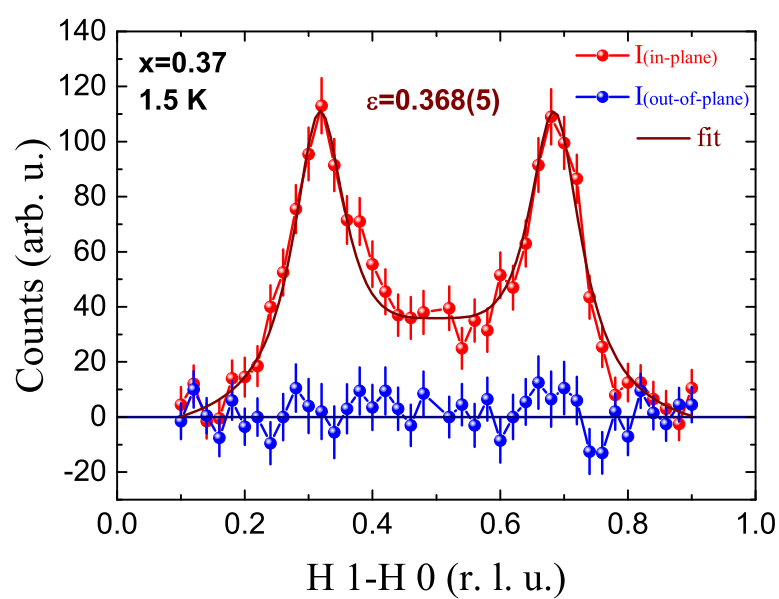

FIG. 2. Characterization of the magnetic correlations in $\mathrm{La}_{1.63} \mathrm{Sr}_{0.37} \mathrm{CoO}_{4}$ by polarized neutrons. All magnetic intensities can be observed in the channel for in-plane magnetically aligned moments. As can be seen, there is also magnetic neutron-scattering intensity around half-integer values of $H$, which is in agreement with a nano phase separation scenario.

dimensional peak. For a two-dimensional Gaussian peak, this volume can be obtained by the amplitude $A$ and the peak widths $\sigma: V=2 \cdot \pi \cdot A \cdot \sigma_{x} \cdot \sigma_{y}$, where $A$ and $\sigma_{x}=\sigma_{y}$ can be obtained from fitting the cut through the two-dimensional Gaussian peak (i.e., the neutron scan) with the function $A$. $\exp \left(-\left(\Delta H^{2} /\left(2 \sigma^{2}\right)\right)\right)$ and with $\sqrt{2} \cdot \sigma=\sigma_{x}$ (because the scan is in a diagonal direction). Thus, we obtain only about $10 \%$ volume fraction for the signal that appears in the $100 \mathrm{~K}-300 \mathrm{~K}$ intensity difference from the longitudinal scanning direction. This volume fraction is only the fraction compared to the $\mathrm{CBCO}$ volume fraction and not the total volume fraction in the sample which has to be even much smaller because of charge balance, which requires the presence of undoped islands. Note that if CSO would appear in these samples, then the CBCO peak should become even broader due to the conversion of parts of the $\mathrm{CBCO}$ regions into CSO regions. Hence, in that case, even the broadening of the half-integer peak could not be entirely attributed to the appearance of a CSO volume fraction, but should also originate from a further increasing short-range character of the $\mathrm{CBCO}$ correlations, i.e., the total $\mathrm{CSO}$ volume fraction should be even distinctly smaller than in our calculations above. Assuming that two satellites also appear for the transversal scanning direction, one can apply a factor of 2 to this value. Nevertheless, this additional signal appears to be only a small fraction compared to more than $\sim 80 \%$ volume fraction of the CBCO phase. If these effects can be only observed for large $L$ values but not for small ones [18], this CSO fraction should be even much smaller.

\section{Synchrotron radiation $x$-ray diffraction}

We also performed off-resonant synchrotron radiation $\mathrm{x}$ ray diffraction experiments to reinvestigate the charge correlations in these cobaltates with a technique that is not sensitive to magnetism. Figure 4(a) first of all shows an $L$ scan through the $\mathrm{CBCO}$ peak position in $\mathrm{La}_{5 / 3} \mathrm{Sr}_{1 / 3} \mathrm{CoO}_{4}$ at $6 \mathrm{~K}$ and $290 \mathrm{~K}$, respectively. As can be seen, charge ordering intensities are strongest for $L=6.5,10.5$, and 14.5 and almost 


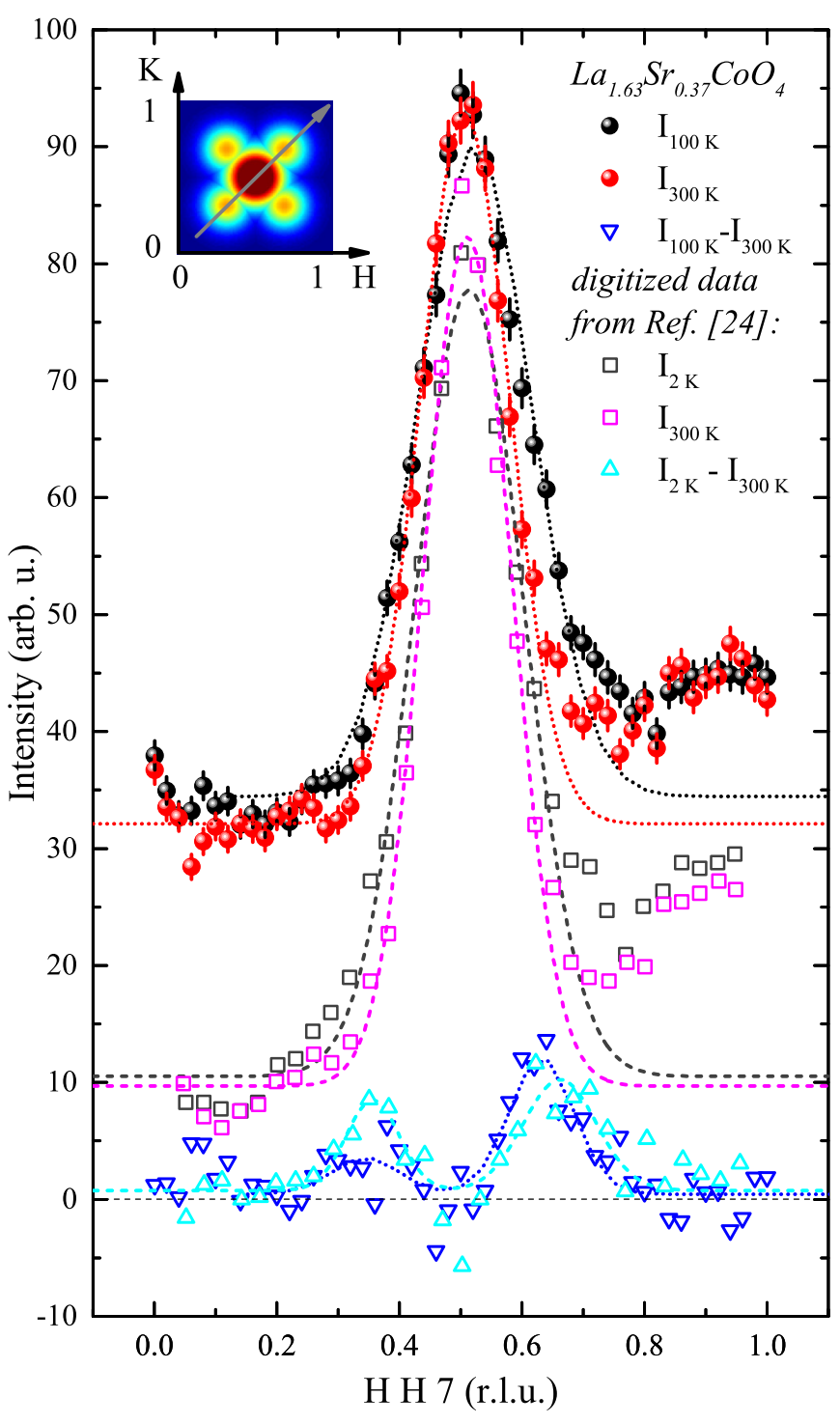

FIG. 3. Non-spin-flip scattering of polarized neutron measurements at the IN20 spectrometer probing the charge correlations in $\mathrm{La}_{1.63} \mathrm{Sr}_{0.37} \mathrm{CoO}_{4}$. Black and red data points denote the lowtemperature data where the intensity of a possible CSO phase should be saturated [24] and the $300 \mathrm{~K}$ data where no possible CSO should appear [24], respectively. We show the raw data without correction for imperfect neutron polarization because we noticed that the flipping ratio became somehow more and more worse with increasing value of $L(2 \Theta)$ such that we decided to use the $100 \mathrm{~K}$ data without correction for imperfect neutron polarization instead. The open square symbols denote the data points digitized from Ref. [24] for the same kind of scan measured at the same instrument. The open triangles denote the differences of low- and high-temperature data and are either indicative for an anomalous peak broadening or for a CSO phase that appears on cooling. The (arrow in the) inset shows the scan within the $H K 7$ plane of reciprocal space (which is not the $H H L$ scattering plane).

temperature independent as noticed already in Refs. [18,19]. The additional sharp peaks for $L=6$ and 12 which appear at lowest temperatures are octahedral tilting reflections, see also Ref. [19]. After identifying the $L$ values for which charge ordering reflections are strongest, we studied the nature of (a)

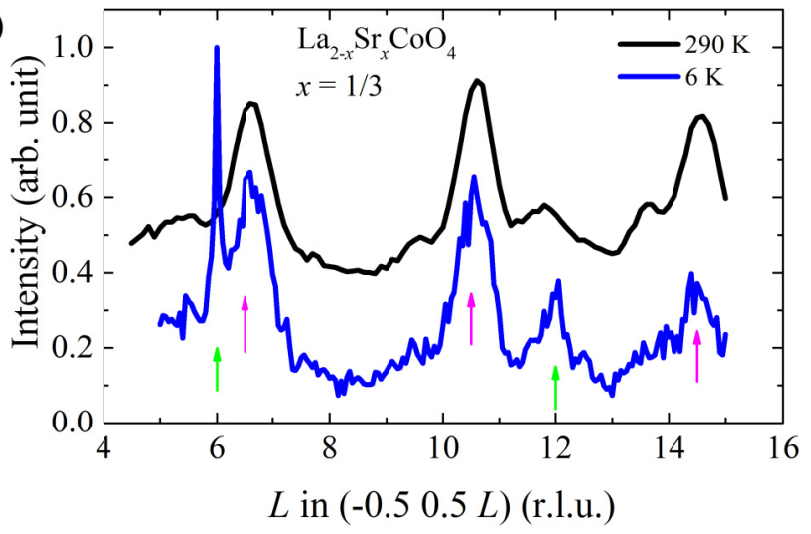

(b)

(c)

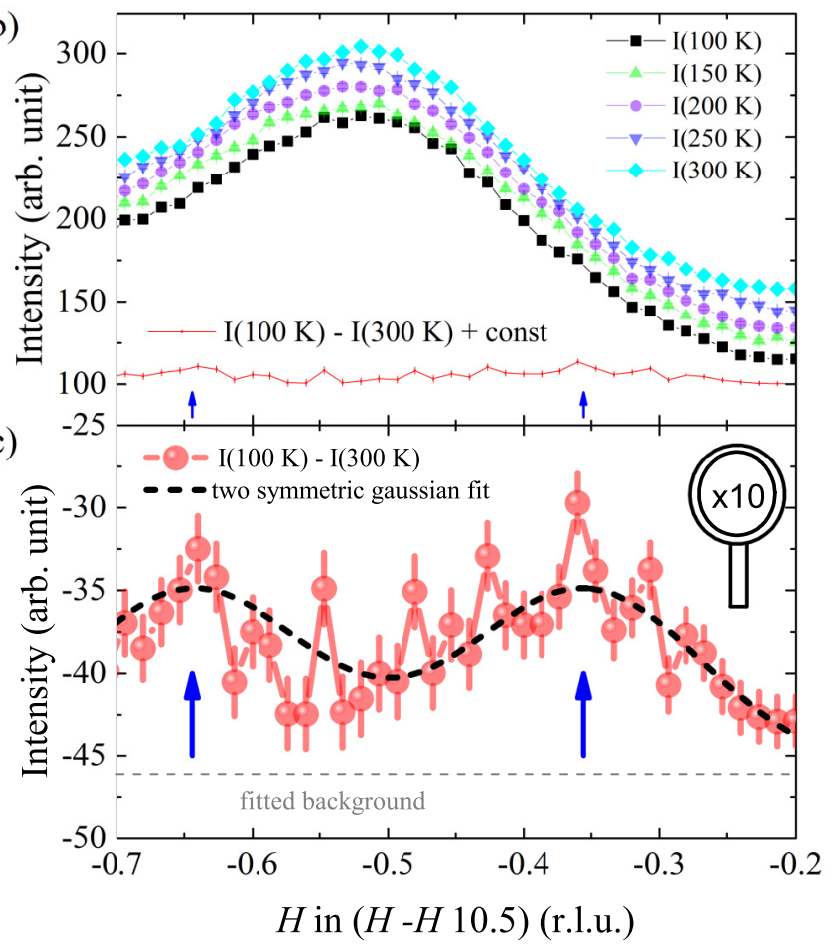

FIG. 4. (a) Synchrotron-radiation diffraction intensities along the $(-0.50 .5 \mathrm{~L})$ direction measured on $\mathrm{La}_{5 / 3} \mathrm{Sr}_{1 / 3} \mathrm{CoO}_{4}$ at 6 and $290 \mathrm{~K}$. Red and green arrows indicate the charge ordering and octahedral tilting peak positions, respectively. (b) Synchrotron radiation diffraction intensities measured on $\mathrm{La}_{5 / 3} \mathrm{Sr}_{1 / 3} \mathrm{CoO}_{4}$ at various temperatures. The intensity difference $I(100 \mathrm{~K})-I(300 \mathrm{~K})$ is shown below the data and also in (c) with $10 \times$ magnification. The blue arrows indicate the expected CSO peak positions.

the underlying charge order. Scans along the $(H-H$ 10.5) direction exhibit a broad peak centered at half-integer values for all temperatures between $100 \mathrm{~K}$ and $300 \mathrm{~K}$, see Fig. 4(b). Whereas the CBCO correlations are clearly apparent, there is no directly apparent evidence for the presence of additional fractions of CSO phases appearing below room temperature. Only when analyzing the intensity differences $I(100 \mathrm{~K})-$ $I(300 \mathrm{~K})$ one can observe some small additional intensities showing up (under $10 \times$ magnification compared to the raw data), see Figs. 4(b) and 4(c). Whether these intensities really originate from CSO correlations or alternatively from an anomalous peak broadening of the CBCO correlations on cooling is unclear. Following the analysis above, in total 
$\left[4 \times I_{\text {shoulder }} /\left(4 \times I_{\text {shoulder }}+I_{\mathrm{CBCO}, 300 K}\right)\right]$ we obtain a fraction of $\sim 15.6 \%$ for any possible CSO phases compared to the volume fraction of the $\mathrm{CBCO}$ phase in $\mathrm{La}_{5 / 3} \mathrm{Sr}_{1 / 3} \mathrm{CoO}_{4}$. This value is roughly consistent with our neutron measurements (that might be less accurate). Note that the total volume fraction of CSO phases has to be even much lower than $\sim 15 \%$ because (in addition to the aforementioned arguments in Sec. III A 1) the presence of yet another phase with undoped islands is also needed for the charge balance of the $\sim 85 \%$ CBCO phase for samples with total hole concentrations far below half-doping. Then, only about $\sim 10 \%$ CSO phase might coexist with $\sim 60 \%$ of the CBCO phase and $\sim 30 \%$ of the phase with undoped islands. Hence, if existent, CSO correlations should not be significant for the appearance of the magnetic excitation spectra in $\mathrm{La}_{5 / 3} \mathrm{Sr}_{1 / 3} \mathrm{CoO}_{4}$ since such a small contribution will be hardly visible in an inelastic neutronscattering experiment. (Note that all three phases are magnetic phases.) In conclusion, the CBCO correlations turn out to be the most robust charge correlations in these cobaltates.

\section{B. X-ray photon correlation spectroscopy}

We have performed XPCS experiments on four different hole concentrations $x=1 / 3,0.37,0.4$, and $1 / 2$. Figure 5 shows the $\mathrm{g}_{2}(\mathrm{t})$ function for different temperatures. The obtained $\mathrm{g}_{2}(\mathrm{t})$ function can be well described by the KWW expression as $\mathrm{g}_{2}(\mathrm{t})=1+A \exp \left[-2(t / \tau)^{\beta}\right]$, where $\tau$ is the relaxation time.

With decreasing hole-doping away from half-doping, the relaxation time of the CBCO decreases rapidly, being already about two times faster for the $x=0.4$ sample and about 10 times faster for the $x=1 / 3$ sample (at $300 \mathrm{~K}$ ), see Fig. 5(b). This change is accompanied by a decrease of the CBCO correlation lengths from about $23 \AA$ for the $x=1 / 2$ sample to about $6 \AA$ for $x=1 / 3$ sample [20]. Hence, the slowly fluctuating $\mathrm{CBCO}$ that we observed in these XPCS experiments is more static (less fluctuating) for hole-doping levels closer to half-doping. This indicates that the charge correlations are most stable for the CBCO order at half-doping. Furthermore, it is interesting to note that the relaxation times $\tau$ follow an almost linear hole-doping $x$ dependence-see inset of Fig. 5(b). The value of $\tau$ extrapolates to zero for $x \rightarrow \sim 0.32$. This is very close to the value where neutron-scattering experiments [18] have observed the breakdown of incommensurate magnetic order in the $\mathrm{La}_{2-x} \mathrm{Sr}_{x} \mathrm{CoO}_{4}$ system for oxygenstoichiometric samples. Hence, incommensurate magnetism within the $\mathrm{La}_{2-x} \mathrm{Sr}_{x} \mathrm{CoO}_{4}$ system below half-doping seems to be inherently connected to the presence of CBCO correlations within a nano phase separation scenario [19].

Another observation is that the $\mathrm{CBCO}$ relaxation time increases with decreasing temperature-see Fig. 5(a). This observation would be consistent with a scenario where the fluctuation of charges decreases on cooling (and maybe also disorder freezes in, which would explain an anomalous CBCO peak-broadening on cooling).

\section{Phase diagram}

Having identified the significant charge correlations in the $\mathrm{La}_{2-x} \mathrm{Sr}_{x} \mathrm{CoO}_{4}$ system, we also studied their temperature
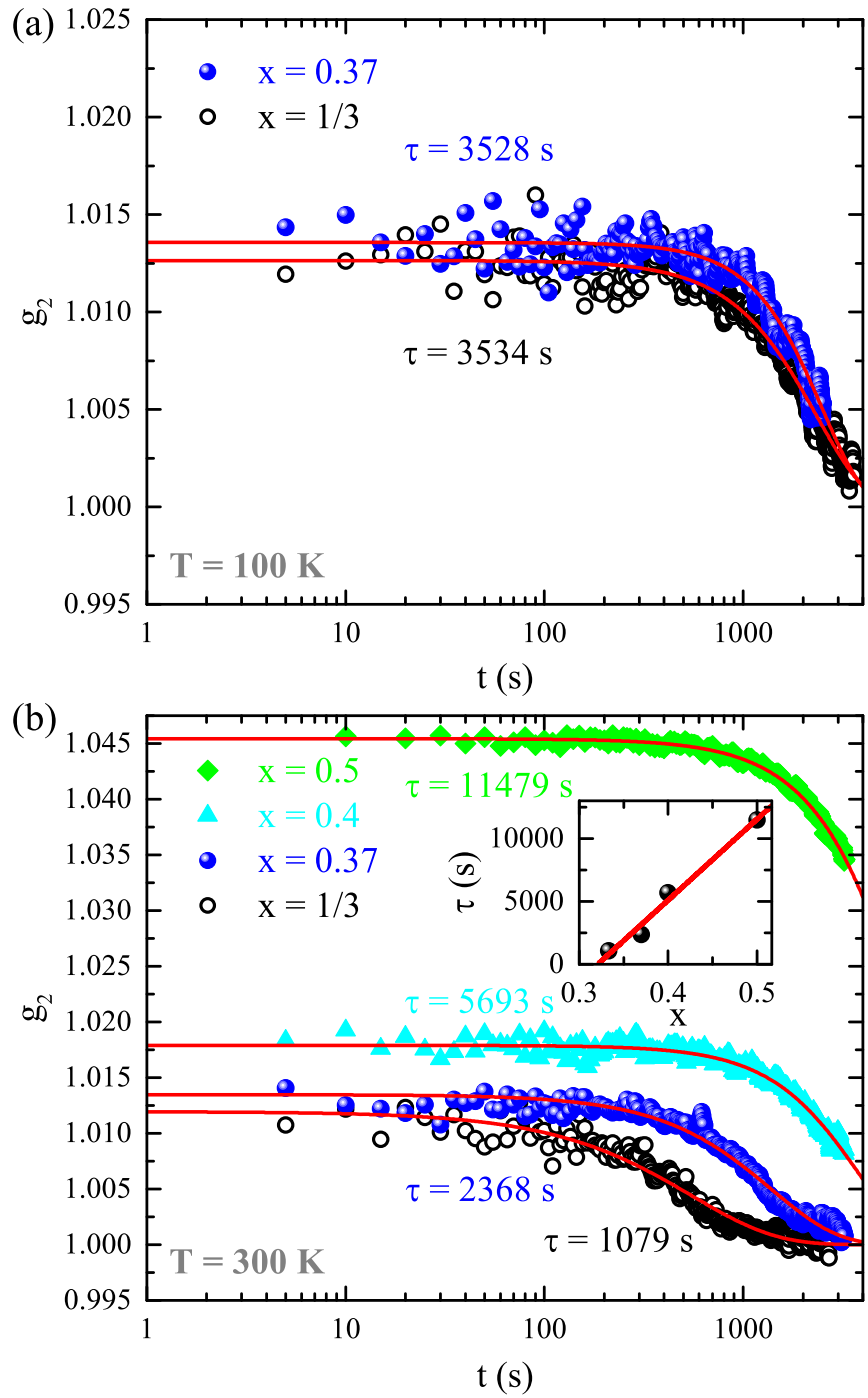

FIG. 5. Normalized intensity autocorrelation function $\mathrm{g}_{2}(\mathrm{t})$ measured at (a) $100 \mathrm{~K}$ and (b) $300 \mathrm{~K}$ for $\mathrm{La}_{2-x} \mathrm{Sr}_{x} \mathrm{CoO}_{4}$ samples with different hole-doping level $x$ as obtained from our XPCS experiments at beamline P10 at DESY. The inset shows the doping dependence of the relaxation time.

dependence within the $\mathrm{La}_{2-x} \mathrm{Sr}_{x} \mathrm{CoO}_{4}$ phase diagram, see Fig. 9. Whether an additional CSO minority phase $(\sim 15 \%$ fraction compared to the $\mathrm{CBCO}$ phase) appears beneath the CBCO phase within the $\mathrm{La}_{2-x} \mathrm{Sr}_{x} \mathrm{CoO}_{4}$ phase diagram or whether there is an anomalous peak broadening on cooling caused by freezing in of disorder, cannot be safely concluded.

\section{Magnetic correlations}

The onset temperatures of (quasistatic) magnetic ordering have been taken from Ref. [30]. Since these values were obtained by neutron scattering which is probing a different timescale, we also reexamined the magnetic phase diagram by means of $\mu \mathrm{SR}$ measurements similar to Ref. [31]. However, we obtain a different magnetic phase diagram with distinctly higher ordering temperatures than reported in Ref. [31]. Figure 6 shows the Fourier transformation of the measured 


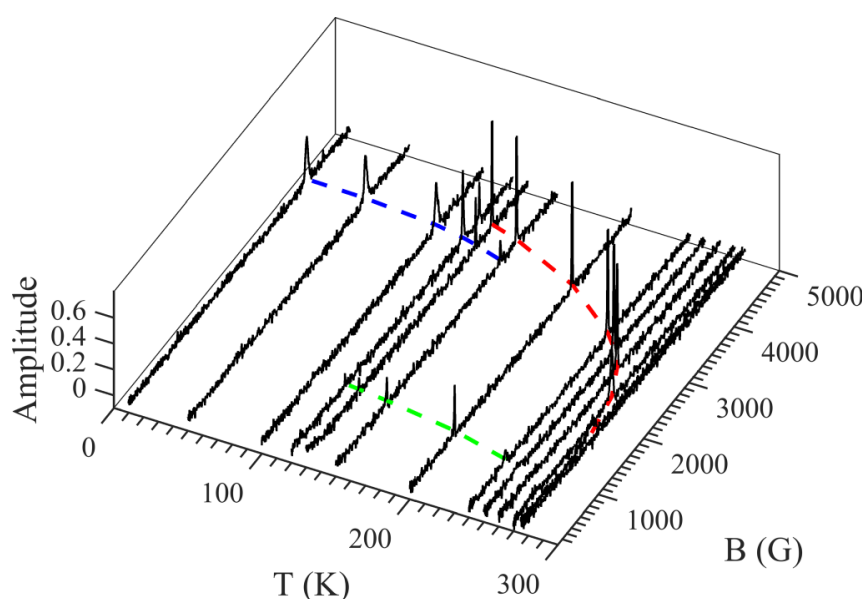

FIG. 6. Fourier transformation of the ZF $\mu$ SR spectra measured at various temperatures for $\mathrm{La}_{2} \mathrm{CoO}_{4}$. The dashed curves are guides to the eye of the evolution of the internal fields.

time spectra at zero field for the $x=0$ sample at various temperatures. Distinct from the previous $\mu$ SR results in the literature [31], spontaneous muon spin precession is already observed below $T_{N} \sim 275 \mathrm{~K}$, which is consistent with our neutron measurements and signals the high quality of the magnetic samples. Two distinct internal fields can be observed below $T_{N}$ which is most likely the consequence of two inequivalent muon sites within the magnetic structure. However, a third component appears below $\sim 150 \mathrm{~K}$ and persists down to the lowest temperature, accompanied by the disappearance of the other two components. This observation reflects the spin reorientation in $\mathrm{La}_{2} \mathrm{CoO}_{4}$ (e.g., see Fig. S3 in Ref. [18]) and the coexistence of the two phases between $100 \mathrm{~K}$ and $150 \mathrm{~K}$. The internal fields amount to about $1285 \mathrm{G}$ and $4260 \mathrm{G}$ at $120 \mathrm{~K}$ for the high-temperature phase, and $4046 \mathrm{G}$ at $10 \mathrm{~K}$ for the low-temperature phase. Furthermore, our other Sr-doped samples $(x=0.1,0.2, \ldots)$ also show distinctly higher magnetic transition temperatures than in literature [31]. Typical transverse-field (TF) measurements are shown in Fig. 7 for the $x=0.2$ sample. Since the internal field is about $4000 \mathrm{G}$ and the muon spin-precession is heavily damped, the application of a small TF (30 G) results in a quickly relaxed signal for muons stopping in the magnetically ordered state. Additionally, a weakly damped oscillating signal for muons stopping in the paramagnetic state is detected with the frequency corresponding to $\gamma_{\mu} B_{\mathrm{TF}}$. The volume fraction of the paramagnetic state corresponds to the amplitude of the oscillating signal. The temperature dependence of the paramagnetic volume fraction is shown in Fig. 7(b). The magnetic ordering onset temperature is defined as the intersection of the linear fitting curve with the dashed curve denoting the fully paramagnetic state. All $\mu$ SR results are summarized in our new phase diagram (magenta dots) shown in Fig. 9. From this phase diagram, it becomes apparent that for the two compositions of $x=0$ and $x=1 / 2$, the $\mu \mathrm{SR}$ measurements reveal similar onset temperatures for magnetic ordering as neutron measurements. This might indicate that only the magnetic order within the undoped phase and within the CBCO phase are able to provide the most stable spin configurations in this system whereas, for other hole-doping

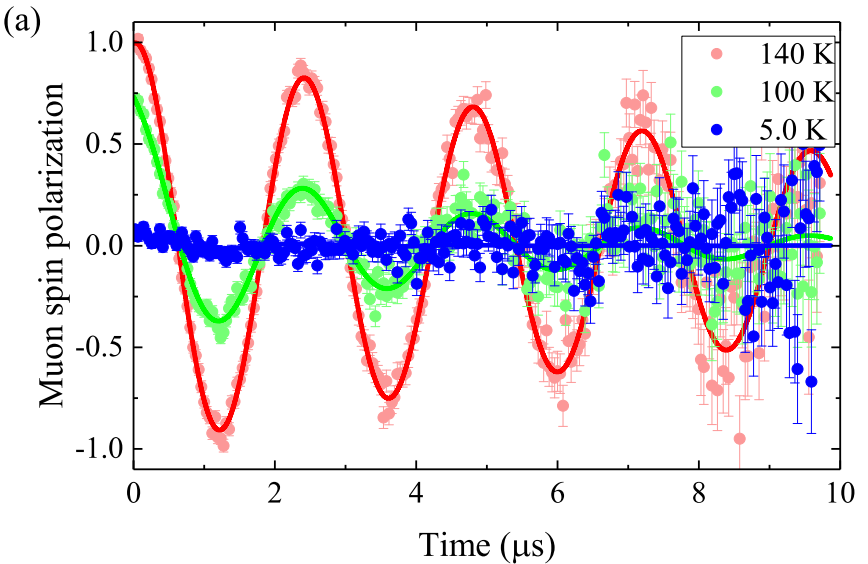

(b)

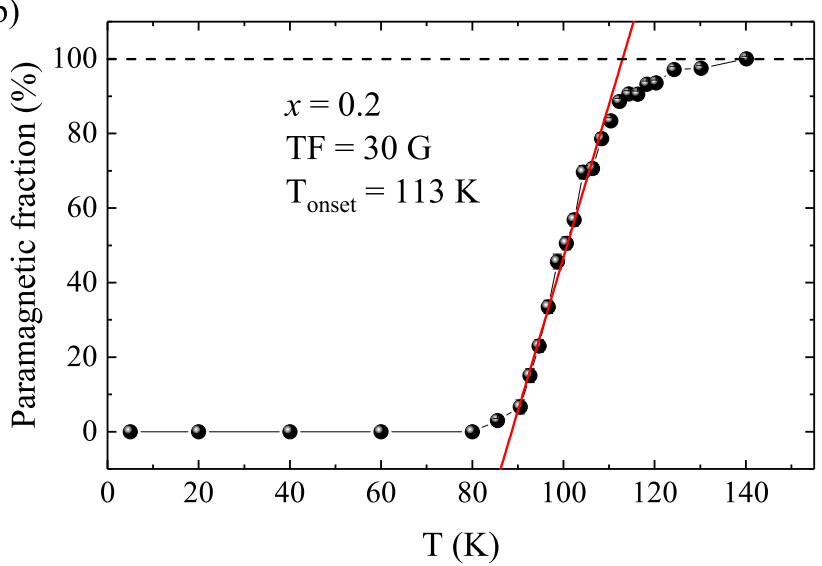

FIG. 7. (a) Typical TF $\mu$ SR spectra measured for $\mathrm{La}_{2-x} \mathrm{Sr}_{x} \mathrm{CoO}_{4}$ in an external field of $30 \mathrm{G}$ for the $x=0.2$ sample. The spectra were fitted with $A(t)=A_{1} \cos \left(\gamma_{\mu} \mathrm{B} t+\varphi\right) \exp \left(-\lambda_{1} t\right)+A_{2} \exp \left(-\lambda_{2} t\right)$ together with the parameter $\alpha$. The solid curves are the fitting results. (b) Temperature dependence of the paramagnetic volume fraction. The magnetic ordering onset temperature is determined by the intersection of the linear fitting curve and the dashed curve.

levels, disorder is present that leads to fluctuations that are getting stronger away from $x=1 / 2$. This is also exactly what we observed in our XPCS experiments - see Fig. 5 - and supports that the $\mathrm{CBCO}$ correlations are the most robust and stable charge correlations in this layered cobaltate system.

\section{Charge order}

The temperature dependence of the half-integer $\mathrm{CBCO}$ peak is shown in Fig. 8 for $\mathrm{La}_{2-x} \mathrm{Sr}_{x} \mathrm{CoO}_{4}$ samples with $x=0.36,0.5$, and 0.6 . The corresponding results are plotted in the phase diagram shown in Fig. 9. The CBCO transition temperature $T_{\mathrm{CBCO}}$ turns out to be independent of $\mathrm{Sr}$ doping, which is in contrast to the behavior of $T_{\mathrm{CBCO}}$ for the isostructural nickelates $\mathrm{La}_{2-x} \mathrm{Sr}_{x} \mathrm{NiO}_{4}$ (LSNO). For these nickelates, the half-doped sample exhibits the highest value of $T_{\mathrm{CBCO}}$ and $T_{\mathrm{CBCO}}$ decreases away from half doping, thus forming a dome shape for the CBCO phase in LSNO [32]. In the cobaltates, the doping independence of $T_{\mathrm{CBCO}}$ points to a local origin of the $\mathrm{CBCO}$ correlations, which is in contrast to the LSNO system where CSO appears on further cooling to lower temperatures. This local origin is most likely caused 


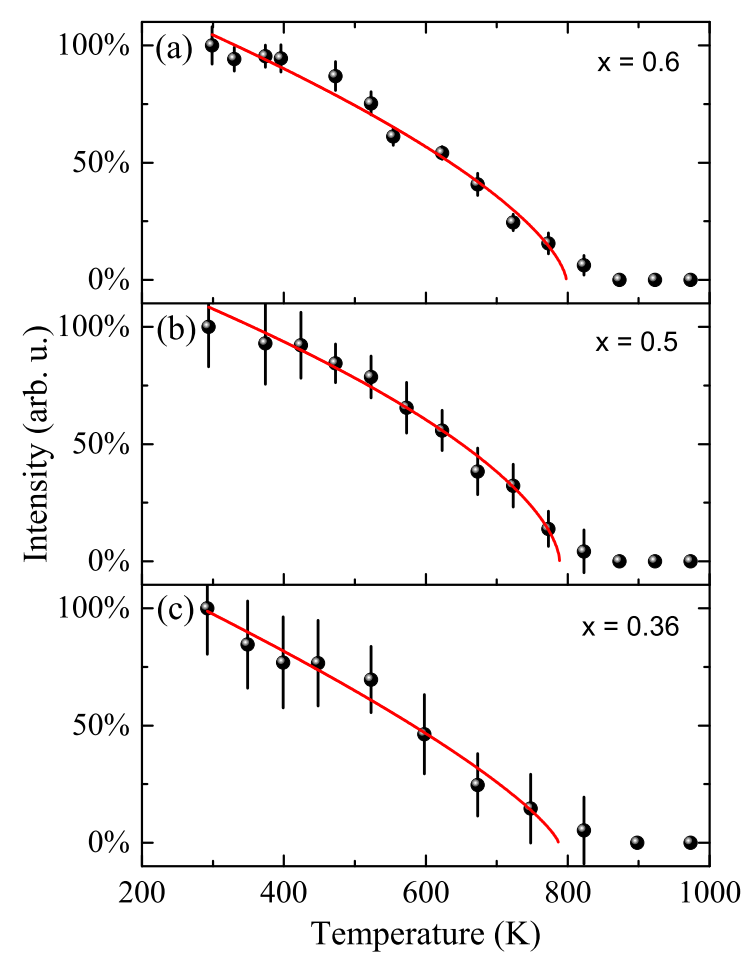

FIG. 8. Temperature dependence of the integrated neutronscattering intensity of the CBCO signal in $\mathrm{La}_{2-x} \mathrm{Sr}_{x} \mathrm{CoO}_{4}$ for $x=$ $0.36,0.5$, and 0.6 observed at half-integer peak positions (2.5 0.50 ) and normalized to the value at room temperature (measured at the IN8 spectrometer).

by the huge difference in the ionic sizes of $\mathrm{Co}^{2+}$ ions in the high-spin state and $\mathrm{Co}^{3+}$ ions in the low-spin state and further strengthened by the insulating properties of these cobaltates. The hopping of electrons from $\mathrm{Co}^{2+}$ to $\mathrm{Co}^{3+}$ sites is hampered due to the spin-blockade mechanism [33]. Hence, also any reorganization of charges into charge stripes is hampered in the quite ionic $\mathrm{La}_{2-x} \mathrm{Sr}_{x} \mathrm{CoO}_{4}$ system even though charges are slowly fluctuating on the timescale of hours. Thus, the cobaltates are different from the LSNO system in which the charges are distributed towards the oxygen ions (ligand holes) and where these charges are able to reorganize into stripes on further cooling to lower temperatures.

\section{Inelastic neutron-scattering experiments}

Furthermore, we have mapped the magnetic excitation spectra of $\mathrm{La}_{1.62} \mathrm{Sr}_{0.38} \mathrm{CoO}_{4}$ and $\mathrm{La}_{1.63} \mathrm{Sr}_{0.37} \mathrm{CoO}_{4}$ with unpolarized and polarized neutrons, respectively. The magnetic incommensurability of these oxygen-stoichiometric samples is very close to the incommensurability of $\mathrm{La}_{5 / 3} \mathrm{Sr}_{1 / 3} \mathrm{CoO}_{4+\delta}$ reported in Ref. [24]. In Fig. 10, the measured magnetic excitation spectra are plotted as a function of energy and momentum transfer. As reported for $\mathrm{La}_{5 / 3} \mathrm{Sr}_{1 / 3} \mathrm{CoO}_{4}[16,19]$ and $\mathrm{La}_{1.6} \mathrm{Sr}_{0.4} \mathrm{CoO}_{4}$ [18], all basic features of an hour-glassshaped magnetic spectrum are reproduced in our samples with intermediate Sr-doping levels, see Figs. 10(a)-10(d). Our polarized neutron study shows that the entire hour-glass dispersion consists of in-plane excitations and that the additional high-energy optical mode above the hour-glass dispersion is an out-of-plane polarized mode.

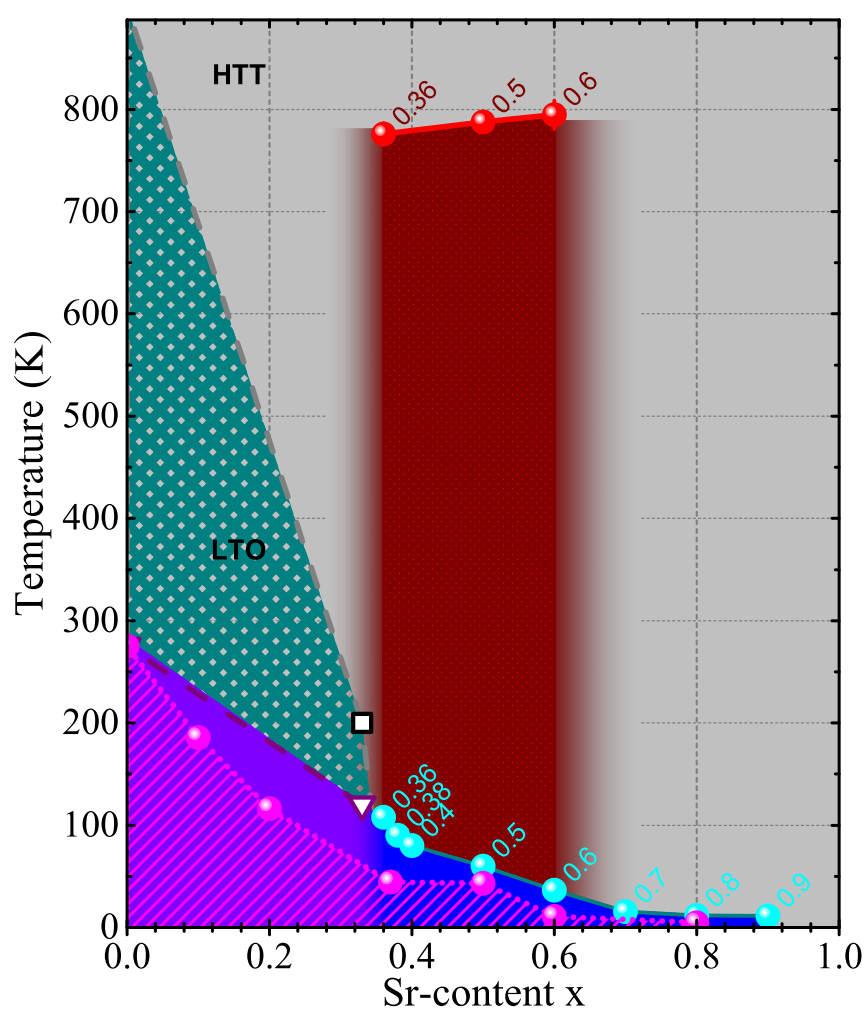

FIG. 9. Our new $\mathrm{La}_{2-x} \mathrm{Sr}_{x} \mathrm{CoO}_{4}$ phase diagram. The structural and magnetic part of the phase diagram was taken from Ref. [30]. Violet and blue areas indicate regimes of quasistatic commensurate and incommensurate magnetic order determined by neutron scattering [30]. The red data points are the $\mathrm{CBCO}$ onset temperatures observed in the present study. Additionally, also the magnetic ordering onset temperatures determined by our $\mu \mathrm{SR}$ measurements are shown (magenta data points). The lower $\mathrm{T}_{N}$ for $x>0$ of $\mu \mathrm{SR}$ compared to neutron values may indicate the different timescales of the experiment with neutrons being the faster probe.

Moreover, three types of scans are schematically indicated in Figs. 10(a) and 10(b) (arrows) that are characteristic for possible spin-anisotropy gaps (scan $A$ ) as well as for lowenergy (scan $B)$ and high energy $(\operatorname{scan} C)$ magnetic excitations within this magnetic excitation spectrum. For these three kinds of scans, we performed a detailed study of their temperature dependence from lowest temperatures up to room temperature. The effect of variation of temperature on the magnetic excitation spectrum of $\mathrm{La}_{1.62} \mathrm{Sr}_{0.38} \mathrm{CoO}_{4}$ was also compared to the corresponding effects in the undoped parent compound $\mathrm{La}_{2} \mathrm{CoO}_{4}$. The magnetic excitation spectrum of $\mathrm{La}_{2} \mathrm{CoO}_{4}$ was studied in Ref. [21] and exhibits a low-energy and a high-energy magnetic mode with an in-plane and an outof-plane spin-anisotropy gap respectively. In Fig. 11(a), the temperature dependence of an energy scan $(\operatorname{scan} A)$ at the planar antiferromagnetic (AFM) wave vector $\mathbf{Q}_{\mathrm{AFM}}$ is shown and provides the essential information about both spin anisotropy gaps in $\mathrm{La}_{2} \mathrm{CoO}_{4}$. As can be seen, the anisotropy gaps in $\mathrm{La}_{2} \mathrm{CoO}_{4}$ decrease on heating to room temperature. Especially, the in-plane anisotropy gap seems to close above about 250 K. In Fig. 11(b), we show the temperature dependence of the same energy scan in $\mathrm{La}_{1.62} \mathrm{Sr}_{0.38} \mathrm{CoO}_{4}$. It is apparent that the corresponding out-of-plane anisotropy gaps are smaller 

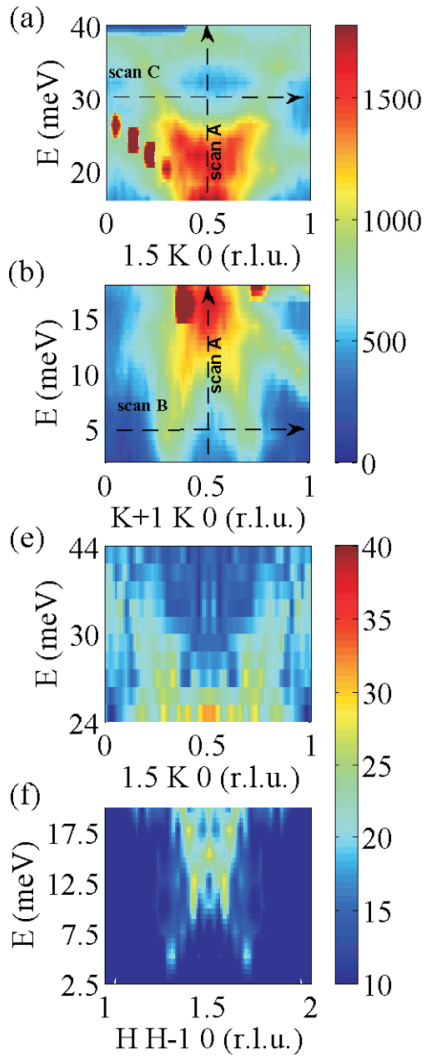
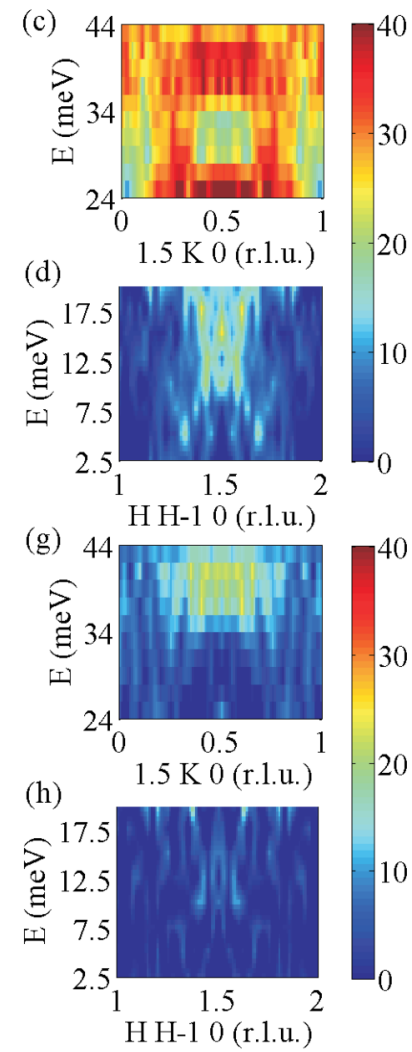

FIG. 10. (a), (b) Magnetic spectrum of $\mathrm{La}_{1.62} \mathrm{Sr}_{0.38} \mathrm{CoO}_{4}$ (intensities are multiplied by $\left.\left(1-\exp \left(-\hbar \omega / k_{B} T\right)\right) /\left|f_{C^{2+}}(\mathbf{Q})\right|^{2}\right)$. Arrows indicate three characteristic scans. (c)-(h) The excitation spectrum of $\mathrm{La}_{1.63} \mathrm{Sr}_{0.37} \mathrm{CoO}_{4}$ is studied with polarized neutrons and the spin-flip scattering cross-sections are shown as a function of energy and momentum transfer. In (c) and (d), the entire magnetic scattering $1 / 2 \cdot\left(\sigma_{x \bar{x}}+\sigma_{\bar{x} x}\right)$ is shown. In (e), (f) and (g), (h) the magnetic scattering $1 / 2 \cdot\left(\sigma_{x \bar{x}}+\sigma_{\bar{x} x}\right)-\sigma_{y \bar{y}}$ and $1 / 2 \cdot\left(\sigma_{x \bar{x}}+\sigma_{\bar{x} x}\right)-\sigma_{z \bar{z}}$ is shown, respectively, with $x$ in direction parallel to the scattering vector $\mathbf{Q}, z$ perpendicular to the scattering plane and $y$ perpendicular to $x$ and $z$. Thus, all magnetic excitations are shown together in (c) and (d), whereas only in-plane and out-of plane excitations are shown in (e), (f) and (g), (h), respectively. For a better comparison with (a) and (b), the data in (c)-(h) was symmetrized since only one side was measured.

in the Sr-doped material. This might be a consequence of the structural changes (altered $\mathrm{CoO}_{6}$ octahedral environment) implied by $\mathrm{Sr}$ doping. Figure 12 displays the temperature dependence of the (a) high-energy and (b) low-energy magnetic excitations that were measured by constant-energy scans at $30 \mathrm{meV}$ (scan C) and $5 \mathrm{meV}$ (scan B), respectively. The corresponding quantitative data analysis is presented in Fig. 13. A third central Gaussian contribution was needed to fit the data of the high-energy scans at elevated temperatures to account for a phonon signal (which has been subtracted in Fig. 12 by fitting another central Gaussian contribution at $K=0.5$ ). The resulting peak intensities, shifts, and peak widths for a fit with two Gaussian contributions (with same peak width) is shown in Fig. 13 and are also compared to the ones in the undoped parent compound $\mathrm{La}_{2} \mathrm{CoO}_{4}$. For $\mathrm{La}_{5 / 3} \mathrm{Sr}_{1 / 3} \mathrm{CoO}_{4}$ a similar analysis without reference materials was made in Ref. [19] up to $100 \mathrm{~K}$ solely. The peak shifts and peak widths that we
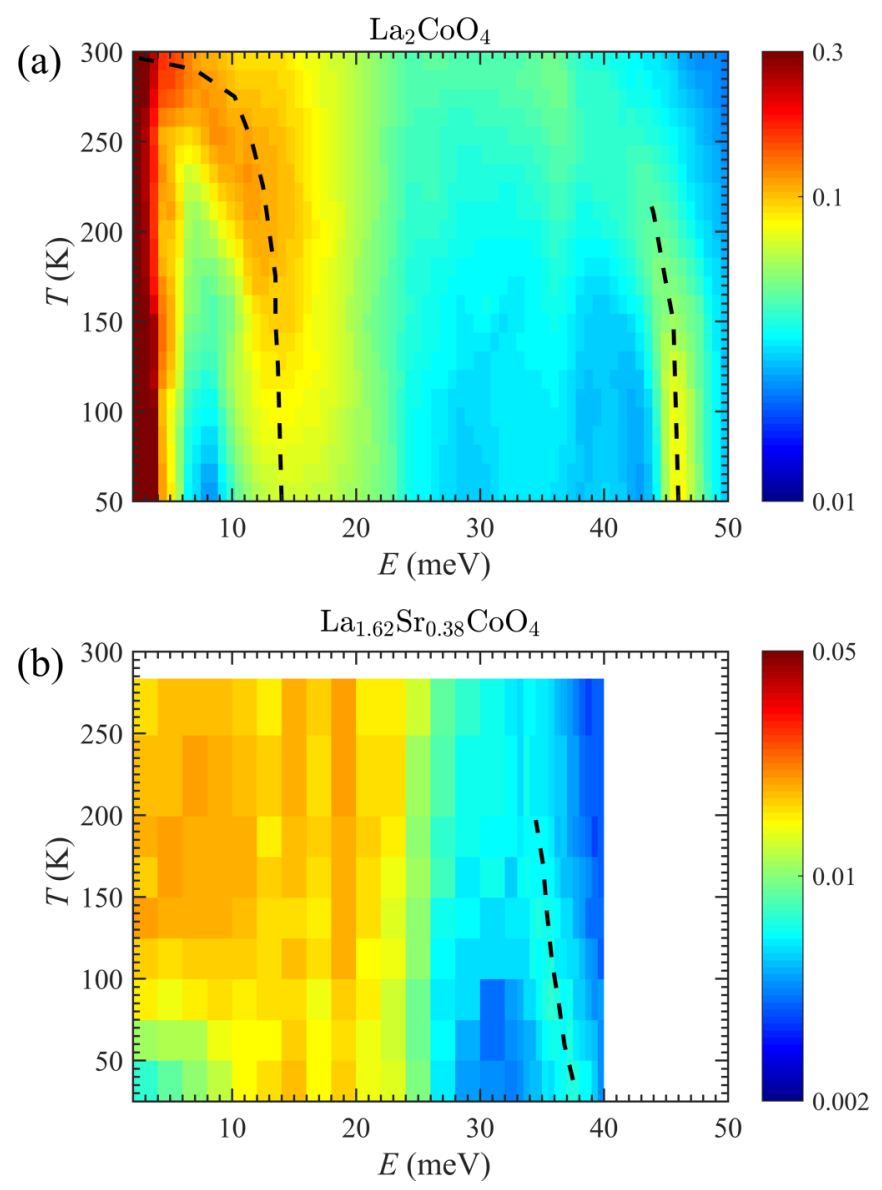

FIG. 11. Temperature dependence of constant-Q scans at $\mathbf{Q}_{\mathrm{AFM}}$ (scan $A$ in Fig. 10) are shown for (a) $\mathrm{La}_{2} \mathrm{CoO}_{4}$ and (b) $\mathrm{La}_{1.62} \mathrm{Sr}_{0.38} \mathrm{CoO}_{4}$. The dashed black lines indicate the peak positions of the in-plane and out-of-plane anisotropy gaps in $\mathrm{La}_{2} \mathrm{CoO}_{4}$. Similar signals at somewhat lower energies can be also found in $\mathrm{La}_{1.62} \mathrm{Sr}_{0.38} \mathrm{CoO}_{4}$.

observe in $\mathrm{La}_{1.62} \mathrm{Sr}_{0.38} \mathrm{CoO}_{4}$ up to room temperature are in accordance with the nano phase separation scenario initially presented in Ref. [19]. For both high- and low-energy excitations, the effect of temperature variation is a peak shift which corresponds to a lowering of the steepness of the dispersion of upper and lower excitation spectra. The peak shift of the highenergy excitations is comparable to the one that we observe in the undoped parent material $\mathrm{La}_{2} \mathrm{CoO}_{4}$ whereas the low-energy magnetic excitations are affected differently. Furthermore, the dynamic correlation length that is reflected by the peak widths is almost temperature invariant. Within such a scenario, the temperature-invariant peak width is the consequence of the limited dynamic correlation length caused by the limited domain size of undoped and hole-doped islands [19].

However, the effect of temperature variation that can be observed for the integrated peak intensities (which were corrected for Bose factor and magnetic form factor) is unexpected. Whereas peak shifts and peak widths behave differently for high-energy and low-energy excitations, the peak intensities exhibit almost the same temperature dependence. This is in contrast to the expected temperature dependence for a conventional phase-separation scenario: For a phase-separated sample consisting of domains of $\mathrm{La}_{2} \mathrm{CoO}_{4}$ 

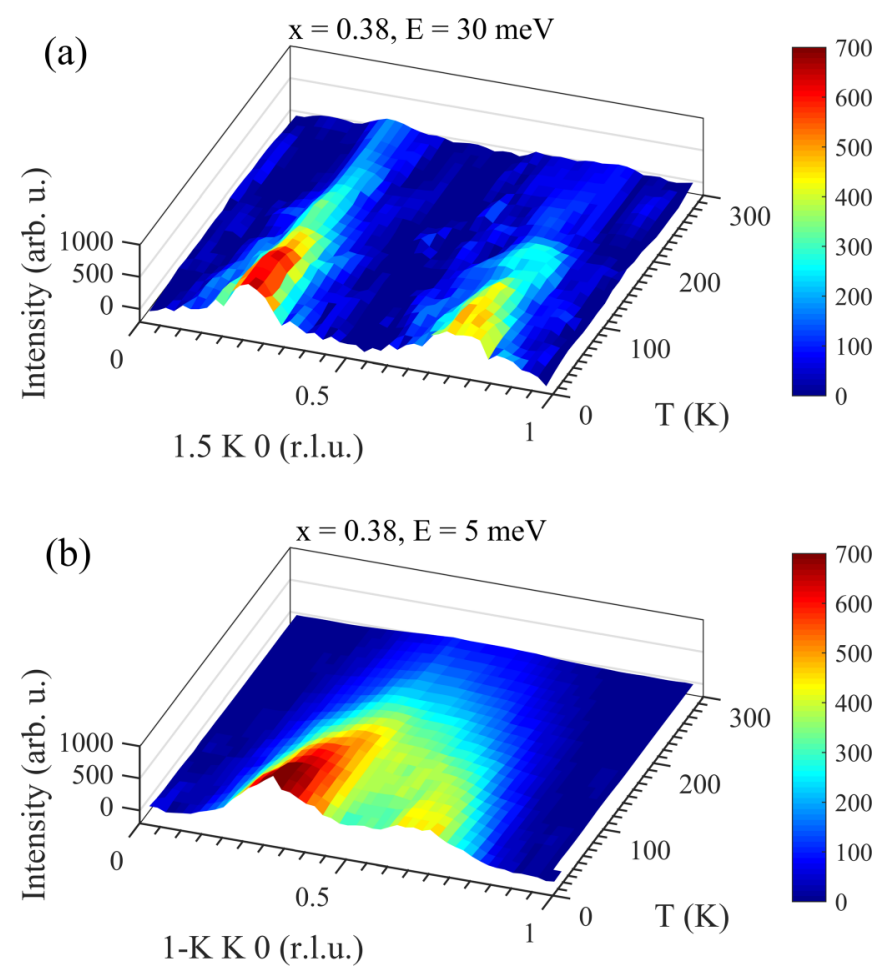

FIG. 12. Temperature dependence of constant-energy scans at (a) $30 \mathrm{meV}$ (scan $C$ in Fig. 10; phonon signal at $K=0.5$ subtracted) and (b) $5 \mathrm{meV}$ (scan $B$ in Fig. 10) are shown for $\mathrm{La}_{1.62} \mathrm{Sr}_{0.38} \mathrm{CoO}_{4}$.

and $\mathrm{La}_{1.5} \mathrm{Sr}_{0.5} \mathrm{CoO}_{4}$ one would expect a different temperature dependence for the magnetic excitations within the undoped and half-doped parts of the sample as is also shown by our comparative measurement of the undoped parent compound $\mathrm{La}_{2} \mathrm{CoO}_{4}$ (green data points in Fig. 13) that is already distinct to the one of $\mathrm{La}_{1.62} \mathrm{Sr}_{0.38} \mathrm{CoO}_{4}$ (red data points in Fig. 13).

\section{DISCUSSION}

That the temperature dependence of the integrated magnetic intensities of high- and low-energy excitations in $\mathrm{La}_{1.62} \mathrm{Sr}_{0.38} \mathrm{CoO}_{4}$ is very similar over a large temperature range up to room temperature shows the difference between nano phase separation and conventional phase separation and that both involved phases cannot be entirely decoupled as suggested by the different temperature dependence of peak shifts and the distinct size of the peak widths for high- and low-energy excitations [19]. Based on this observation, one can understand some features of the magnetic excitation spectra starting from the ideal $\mathrm{CBCO} \mathrm{La}_{1.5} \mathrm{Sr}_{0.5} \mathrm{CoO}_{4}$ material: For $x=1 / 2$, all transverse spin excitations are equivalent independent whether one follows an outwards- or inwardsdispersing branch, (cf. Fig. 14 for $x=1 / 2$ ). Indeed, the experiment also shows very similar intensities for outwardsand inwards-dispersing branches in $\mathrm{La}_{1.5} \mathrm{Sr}_{0.5} \mathrm{CoO}_{4}$ [22]. But, in Fig. 14 it is also shown what happens below half-doping, according to our nano phase separation scenario when undoped islands (red regions) are inserted into a CBCO matrix. Irrespective of the incommensurate propagation vector, the case is not anymore symmetric for outwards- and inwardsdispersing branches (note that dynamic correlation lengths

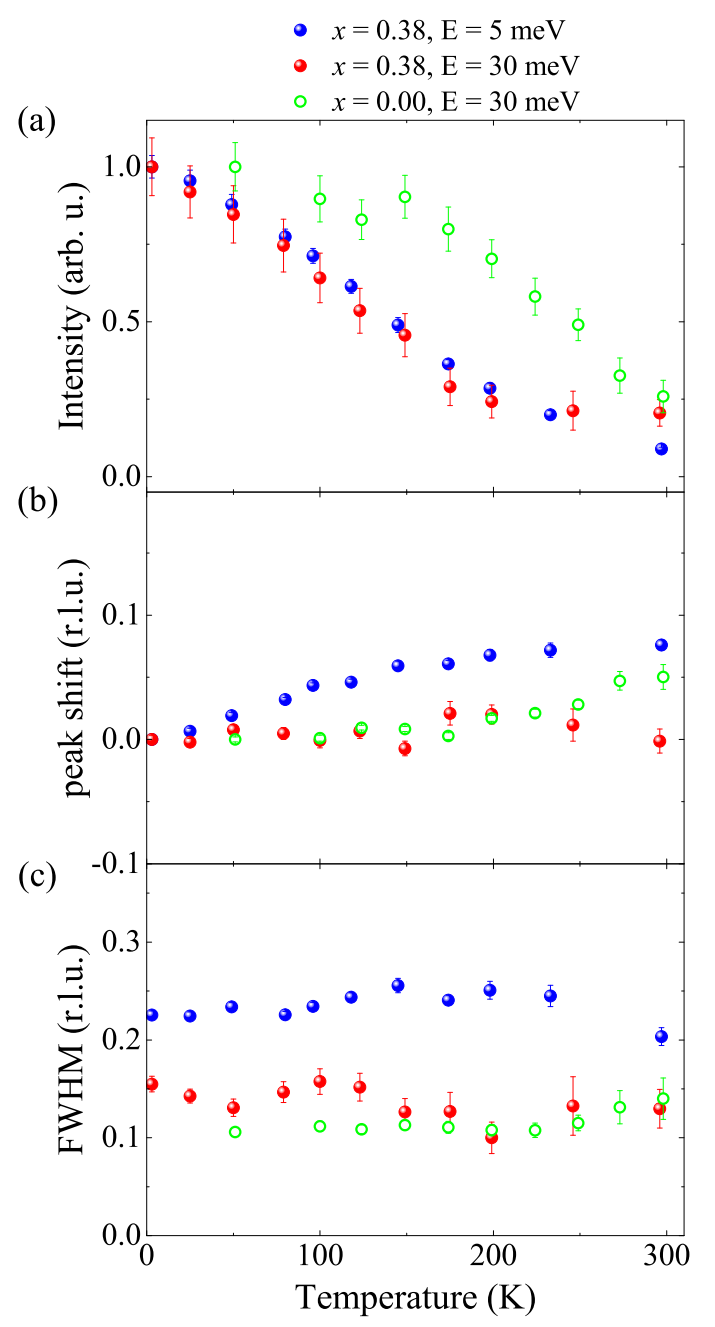

FIG. 13. For high-energy (red) and low-energy (blue) excitations in $\mathrm{La}_{1.62} \mathrm{Sr}_{0.38} \mathrm{CoO}_{4}$ the temperature dependence of (a) the integrated peak intensities corrected for Bose factor and magnetic form factor, (b) the peak positional shifts compared to the peak position at base temperature, and (c) the peak widths are shown for constant-energy scansat $5 \mathrm{meV}$ or $30 \mathrm{meV}$. Additionally, the fitting values for the corresponding scan at $30 \mathrm{meV}$ in the $\mathrm{La}_{2} \mathrm{CoO}_{4}$ reference sample are shown.

are short and that spins are predominantly antiferromagnetically coupled within an undoped island). For $\mathbf{Q}$ vectors of inwards-dispersing branches, the transverse spin excitations will preserve a predominantly antiferromagnetic alignment of the spins. This is energetically favorable since the nn exchange interaction $|J|$ is much bigger than $\left|J^{\prime}\right|$. In contrast to that, the transverse spin excitations in undoped islands would break the antiferromagnetic symmetry for $\mathbf{Q}$ vectors of the outwardsdispersing branches which is energetically extremely unfavorable due to the huge size of the nn exchange interaction within these undoped islands. Hence, the quite symmetric situation as in $\mathrm{La}_{1.5} \mathrm{Sr}_{0.5} \mathrm{CoO}_{4}$ is completely broken once nanometersized undoped islands with strong antiferromagnetic nn interactions are inserted, and the suppression of the outwardsdispersing branches in hour-glass spectra is a consequence of the presence of nano phase separation which is distinct from conventional phase separation since the observed magnetic 


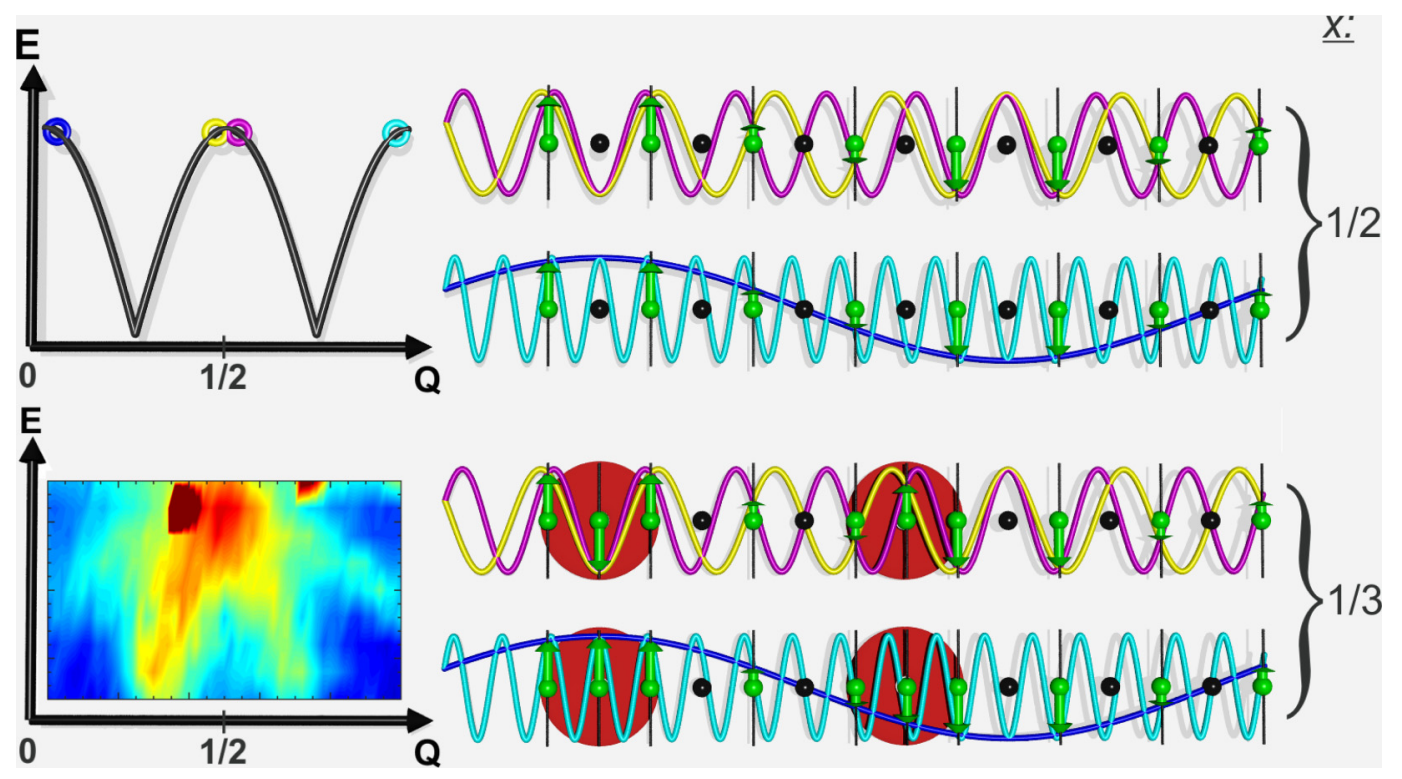

FIG. 14. Schematic figure showing the transverse spin excitations for momentum transfers $\mathbf{Q}$ of inwards- (yellow and magenta) and outwards- (light and dark blue) dispersing branches in $\mathrm{La}_{2-x} \mathrm{Sr}_{x} \mathrm{CoO}_{4}$ for $x=1 / 2$ (upper two representations) and $\sim 1 / 3$ (lower two representations), respectively. For a quarter-integer propagation vector, the spins would exhibit an antiferromagnetic orientation for $x=1 / 2$ due to the presence of AFM next-nearest-neighboring (nnn) exchange interactions $J^{\prime}$. Then, independent of outwards- or inwards-dispersing directions, the alignment of spins of nnn Co-ions becomes less antiparallel (i.e., more ferromagnetic) away from the quarter-integer propagation vector. However, this symmetric situation is broken, when undoped islands are introduced into the system within a nano phase separation scenario $(x \sim 1 / 3)$ : For $\mathbf{Q}$ vectors corresponding to an outwards dispersion, the spins would be additionally more ferromagnetically aligned within the undoped islands that host the strongest nearest-neighboring (nn) exchange interactions $J$. This is an unfavorable situation regarding the huge nn exchange interactions $|J| \gg\left|J^{\prime}\right|$ and in contrast to an inwards-dispersing branch where the spins within an undoped island still couple predominantly antiferromagnetically. Thus, nano phase separation naturally explains the breaking of the initially rather symmetric situation for inwards- and outwards-dispersing branches. Note that the momentum transfer $\mathbf{Q}$ (left panels) is along the [110]-direction whereas the real space moment configurations (right panels) show the evolution of the spins along [100] or [010] direction only and have to be repeated correspondingly along the perpendicular direction [010] or [100], respectively.

excitation spectra are not a simple superposition of the spectra of both phases that are involved.

\section{CONCLUSION}

Concluding, charge modulations with quarter- (1/4-) and third-integer (1/3-) propagation vectors belong to the most robust charge correlations in cuprates and nickelates with $\mathrm{K}_{2} \mathrm{NiF}_{4}$ structures, whereas for the next system in this rowthe cobaltates-charge modulations with half-integer $(1 / 2)$ propagation vectors seem to be the most robust charge correlations even far below half-doping as indicated by synchrotron radiation single crystal $\mathrm{x}$-ray diffraction, XPCS, and $\mu \mathrm{SR}$ experiments. Below half-doping, the emergence of undoped islands within a nano phase separation scenario is required for charge balance. Whether some of these islands might also have a stripe-like appearance (with max. $\sim 15 \%$ volume fraction compared to the $\mathrm{CBCO}$ volume fraction or $\sim 10 \%$ in total for this possible scenario) is yet unclear. Alternatively, the $\mathrm{CBCO}$ correlations could also exhibit an anomalous peak broadening on cooling. Moreover, the high hole-doping invariant $\mathrm{CBCO}$ ordering temperatures that have been observed in neutron-scattering experiments point to a more local origin of the charge correlations in these rather ionic cobaltates, thus being distinct from the more covalent nickelates with ligand holes being able to reorganize into charge stripes at lower temperatures. XPCS experiments reveal a slowly fluctuating character of these charges which are connected to the emergence of hour-glass magnetic spectra.

For the suppression of the outwards-dispersing branches in hour-glass spectra, which is one of the most characteristic features of these spectra, a microscopic understanding was missing. In the past, disorder was connected to this suppression [16,17]. Our systematic measurements of the magnetic excitations in $\mathrm{La}_{2-x} \mathrm{Sr}_{x} \mathrm{CoO}_{4}(0 \leqslant x \leqslant 1 / 2)$ reveal a similar temperature dependence of high- and low-energy magnetic excitations within an hour-glass spectrum which would not be expected for conventional phase separation. Also, the suppression of the outwards-dispersing branches would not be expected for conventional phase separation where the spectrum would be a kind of superposition of the spectra of $\mathrm{La}_{1.5} \mathrm{Sr}_{0.5} \mathrm{CoO}_{4}$ and $\mathrm{La}_{2} \mathrm{CoO}_{4}$. These observations indicate that high- and low-energy excitations are not entirely decoupled in a nano phase separation scenario and give rise to a microscopic scenario that naturally explains the suppression of the outwards-dispersing branches in hour-glass spectra. This scenario is based on nano phase separation where undoped islands host strongly 
antiferromagnetically coupled nearest-neighboring spins which induces a breaking of the initially symmetric situation for inwards and outwards [i.e., towards or away from the planar antiferromagnetic wave-vector $(1 / 21 / 20)]$ dispersing branches. Our findings unveil the importance of nano phase separation for the emergence of hour-glass spectra in cobaltates and show that conventional phase separation and nano phase separation have to be considered as qualitatively distinct phenomena.

Experimental data are available from the Institut LaueLangevin (ILL) [34-38].

\section{ACKNOWLEDGMENTS}

The research in Dresden is supported by the Deutsche Forschungsgemeinschaft through Grant No. 320571839. We thank F. Marchal for technical support. We thank F. Westermeier for technical assistance. We thank A. Ivanov for support. G.R. acknowledges the financial support by the National Research Foundation of Korea (NRF) funded by the Ministry of Science and ICT (No. 2016K1A4A4A01922028). We acknowledge support from the Max Planck-POSTECHHsinchu Center for Complex Phase Materials. We thank M. Rotter for helpful discussions.
[1] J. M. Tranquada, H. Woo, T. G. Perring, H. Goka, G. D. Gu, G. Xu, M. Fujita, and K. Yamada, Nature 429, 534 (2004).

[2] M. Matsuda, M. Fujita, S. Wakimoto, J. A. Fernandez-Baca, J. M. Tranquada, and K. Yamada, Phys. Rev. Lett. 101, 197001 (2008).

[3] M. Arai, T. Nishijima, Y. Endoh, T. Egami, S. Tajima, K. Tomimoto, Y. Shiohara, M. Takahashi, A. Garrett, and S. M. Bennington, Phys. Rev. Lett. 83, 608 (1999).

[4] P. Bourges, Y. Sidis, H. F. Fong, L. P. Regnault, J. Bossy, A. Ivanov, and B. Keimer, Science 288, 1234 (2000).

[5] N. B. Christensen, D. F. McMorrow, H. M. Rønnow, B. Lake, S. M. Hayden, G. Aeppli, T. G. Perring, M. Mangkorntong, M. Nohara, and H. Takagi, Phys. Rev. Lett. 93, 147002 (2004).

[6] S. M. Hayden, H. A. Mook, P. Dai, T. G. Perring, and F. Dogan, Nature 429, 531 (2004).

[7] B. Vignolle, S. M. Hayden, D. F. McMorrow, H. M. Rønnow, B. Lake, C. D. Frost, and T. G. Perring, Nat. Phys. 3, 163 (2007).

[8] G. Xu, G. D. Gu, M. Hücker, B. Fauqué, T. G. Perring, L. P. Regnault, and J. M. Tranquada, Nat. Phys. 5, 642 (2009).

[9] O. J. Lipscombe, B. Vignolle, T. G. Perring, C. D. Frost, and S. M. Hayden, Phys. Rev. Lett. 102, 167002 (2009).

[10] V. Hinkov, P. Bourges, S. Pailhès, Y. Sidis, A. Ivanov, C. D. Frost, T. G. Perring, C. T. Lin, D. P. Chen, and B. Keimer, Nature Physics 3, 780 (2007).

[11] M. Fujita, H. Hiraka, M. Matsuda, M. Matsuura, J. M. Tranquada, S. Wakimoto, G. Xu, and K. Yamada, J. Phys. Soc. Jpn. 81, 011007 (2012).

[12] I. Eremin, D. K. Morr, A. V. Chubukov, K. H. Bennemann, and M. R. Norman, Phys. Rev. Lett. 94, 147001 (2005).

[13] M. Vojta, T. Vojta, and R. K. Kaul, Phys. Rev. Lett. 97, 097001 (2006).

[14] G. Seibold and J. Lorenzana, Phys. Rev. Lett. 94, 107006 (2005).

[15] B. M. Andersen, S. Graser, and P. J. Hirschfeld, Phys. Rev. Lett. 105, 147002 (2010).

[16] A. T. Boothroyd, P. Babkevich, D. Prabhakaran, and P. G. Freeman, Nature 471, 341 (2011).

[17] E. C. Andrade and M. Vojta, Phys. Rev. Lett. 109, 147201 (2012).

[18] Y. Drees, D. Lamago, A. Piovano, and A. C. Komarek, Nat. Commun. 4, 2449 (2013).

[19] Y. Drees, Z. W. Li, A. Ricci, M. Rotter, W. Schmidt, D. Lamago, O. Sobolev, U. Rütt, O. Gutowski, M. Sprung, A. Piovano, J. P. Castellan, and A. C. Komarek, Nat. Commun. 5, 5731 (2014).
[20] H. Guo, W. Schmidt, L. H. Tjeng, and A. C. Komarek, Phys. Status Solidii RRL 9, 580 (2015).

[21] P. Babkevich, D. Prabhakaran, C. D. Frost, and A. T. Boothroyd, Phys. Rev. B 82, 184425 (2010).

[22] L. M. Helme, A. T. Boothroyd, R. Coldea, D. Prabhakaran, C. D. Frost, D. A. Keen, L. P. Regnault, P. G. Freeman, M. Enderle, and J. Kulda, Phys. Rev. B 80, 134414 (2009).

[23] Z. W. Li, Y. Drees, A. Ricci, D. Lamago, A. Piovano, M. Rotter, W. Schmidt, O. Sobolev, U. Rütt, O. Gutowski, M. Sprung, J. P. Castellan, L. H. Tjeng, and A. C. Komarek, J. Supercond. Nov. Magn. 29, 727 (2016).

[24] P. Babkevich, P. G. Freeman, M. Enderle, D. Prabhakaran, and A. T. Boothroyd, Nat. Commun. 7, 11632 (2016).

[25] G. Campi, A. Bianconi, N. Poccia, G. Bianconi, L. Barba, G. Arrighetti, D. Innocenti, J. Karpinski, N. D. Zhigadlo, S. M. Kazakov, M. Burghammer, M. v. Zimmermann, M. Sprung, and A. Ricci, Nature 525, 359 (2015).

[26] R. H. Yuan, T. Dong, Y. J. Song, P. Zheng, G. F. Chen, J. P. Hu, J. Q. Li, and N. L. Wang, Sci. Rep. 2, 221 (2012).

[27] A. Ricci, N. Poccia, B. Joseph, D. Innocenti, G. Campi, A. Zozulya, F. Westermeier, A. Schavkan, F. Coneri, A. Bianconi, H. Takeya, Y. Mizuguchi, Y. Takano, T. Mizokawa, M. Sprung, and N. L. Saini, Phys. Rev. B 91, 020503(R) (2015).

[28] P. Giraldo-Gallo, Y. Zhang, C. Parra, H. Manoharan, M. Beasley, T. Geballe, M. Kramer, and I. Fisher, Nat. Commun. 6, 8231 (2015).

[29] A. Suter and B. M. Wojek, Phys. Procedia 30, 69 (2012).

[30] Z. W. Li, Y. Drees, C. Y. Kuo, H. Guo, A. Ricci, D. Lamago, O. Sobolev, U. Rütt, O. Gutowski, T. W. Pi, A. Piovano, W. Schmidt, K. Mogare, Z. Hu, L. H. Tjeng, and A. C. Komarek, Sci. Rep. 6, 25117 (2016).

[31] R. C. Williams, F. Xiao, T. Lancaster, R. De Renzi, G. Allodi, S. Bordignon, P. G. Freeman, F. L. Pratt, S. R. Giblin, J. S. Möller, S. J. Blundell, A. T. Boothroyd, and D. Prabhakaran, Phys. Rev. B 93, 140406(R) (2016).

[32] R. Kajimoto, K. Ishizaka, H. Yoshizawa, and Y. Tokura, Phys. Rev. B 67, 014511 (2003).

[33] C. F. Chang, Z. Hu, H. Wu, T. Burnus, N. Hollmann, M. Benomar, T. Lorenz, A. Tanaka, H.-J. Lin, H. H. Hsieh, C. T. Chen, and L. H. Tjeng, Phys. Rev. Lett. 102, 116401 (2009).

[34] H. Guo, A. Piovano, and A. C. Komarek, Temperature Dependence of Magnetic Excitations in Cobaltates (Institut LaueLangevin (ILL), 2015), doi:10.5291/ILL-DATA.DIR-140. 
[35] H. Guo, Z. W. Li, A. Piovano, W. Schmidt, and A. C. Komarek, Polarization Analysis of Hour-Glass-Shaped Magnetic Excitations in $\mathrm{La}_{2-x} \mathrm{Sr}_{x} \mathrm{CoO}_{4}$ (Institut Laue-Langevin (ILL), 2015), doi:10.5291/ILL-DATA.4-01-1422.

[36] H. Guo, W. Schmidt, and A. C. Komarek, Nano Phase Separation in Oxygen Ordered and Disordered Nickelates and Cuprates (Institut Laue-Langevin (ILL), 2016), doi:10.5291/ILL-DATA.4-01-1522.
[37] Z. W. Li, H. Guo, A. Piovano, W. Schmidt, and A. C. Komarek, High-Temperature Study of Charge Correlations in Layered Cobalt Oxides (Institut Laue-Langevin (ILL), 2015), doi:10.5291/ILL-DATA.5-11-412.

[38] Z. W. Li, H. Guo, A. Piovano, and A. C. Komarek, Study of the Temperature Dependence of the Hour-Glass Dispersion in Cobaltates (Institut Laue-Langevin (ILL), 2014), doi:10.5291/ILL-DATA.4-01-1368. 